\title{
Fluoride-Releasing Effect of a Modified Resin Denture Containing S-PRG Fillers on Salivary Fluoride Retention: A Randomized Clinical Study
}

\author{
Kritirat Kiatsirirote $^{\mathrm{a}}$ Thanya Sitthisettapong ${ }^{\mathrm{a}}$ Prathip Phantumvanit ${ }^{\mathrm{a}}$ \\ Daniel C.N. Chan ${ }^{b}$ \\ ${ }^{a}$ Faculty of Dentistry, Thammasat University, Pathumthani, Thailand; ${ }^{b}$ Department of Restorative Dentistry, \\ School of Dentistry, University of Washington, Seattle, WA, USA
}

\section{Keywords}

Caries prevention - Clinical trial · Resin denture base ·

S-PRG fillers · Saliva fluoride

\begin{abstract}
This study aimed to evaluate the clinical effect of a resin denture base containing 20wt\% surface prereacted glass ionomer (S-PRG) fillers on saliva fluoride concentrations as a surrogate evaluation of caries control. The study was a doubleblind randomized clinical trial with a total of 110 participants aged 35-60 years who wore a removable partial resin denture for 1.5 years. Subjects were randomly assigned to receive a partial resin denture fabricated either with or without 20wt\% S-PRG fillers ( $n=55$ per group). After 14 days of wearing the dentures, 5,000 ppm fluoride gel was applied nightly on both sides of the denture before storing it in a denture box overnight every night for 1.5 years. The salivary fluoride concentration was measured at baseline, on days 1, 14, and 15 , and at 3 months and 1.5 years of wearing the denture. $A$ significant increase in salivary fluoride concentration in the S-PRG denture group was observed from baseline to day 1, followed by a significant decrease to an approximate baseline level. After recharge with 5,000 ppm fluoride gel, salivary
\end{abstract}

\section{KARGER}

(c) 2018 S. Karger AG, Basel

E-Mail karger@karger.com

www.karger.com/cre fluoride increased markedly on day 15 and remained elevated at month 3 through to 1.5 years. The resin denture base containing $20 \mathrm{wt} \%$ S-PRG fillers demonstrated an initial fluoride release that increased saliva fluoride concentrations, but the fluoride release from within the material was short lived. The long-term effect of sustained fluoride release from the denture when combined with a regular fluoride recharge regimen may be beneficial for caries prevention.

(c) 2018 S. Karger AG, Basel

\section{Introduction}

Resin dentures are a typical option for people who exhibit partial tooth loss, especially in older populations due to economic factors. This type of denture can replace multiple teeth and is characterized by the ease of fabrication and low cost. However, resin dentures require wide coverage over surrounding abutment teeth, which subsequently are more likely than any other teeth to be affected by caries. As a means of preventing caries, the den-

ClinicalTrials.gov registration No. NCT 02460562.

Dr. Kritirat Kiatsirirote

Faculty of Dentistry, Thammasat University 99 Moo 18 Paholyothin Road

Khlongluang, Pahtumthani 12121 (Thailand)

E-Mail kkritirat@yahoo.com 


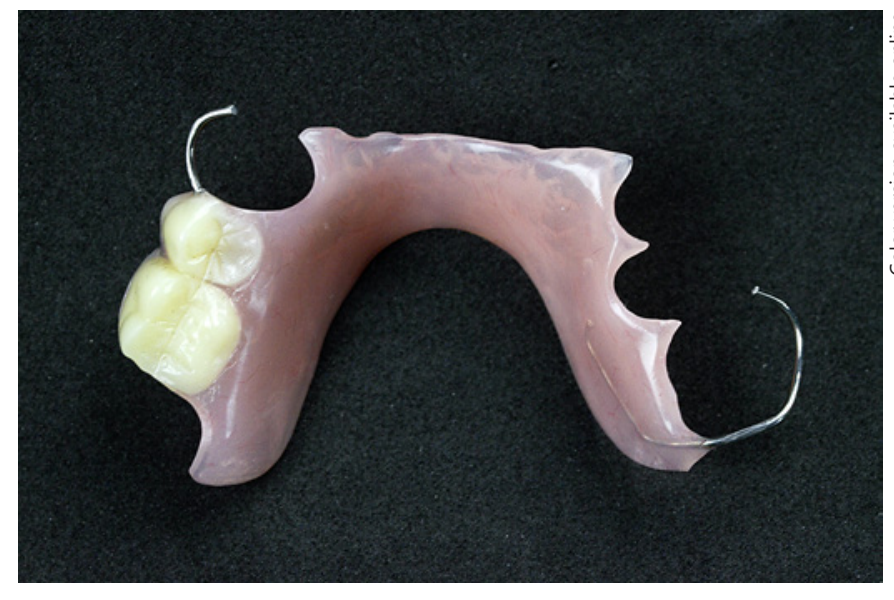

Fig. 1. Upper partial resin denture without S-PRG fillers.

ture wearer must attend to oral care and fluoride delivery using a variety of recommended methods [Han et al., 2002]. One such method is by the use of fluoride-containing dentures given their ability to maintain a therapeutic concentration of fluoride over extended periods of time. Recently, surface prereacted glass-ionomer (SPRG) filler has been introduced. It is a new type of biological material which is being used as filling material in the formulation of dental products, partly because of its ability to release and recharge fluoride. The fluoride in turn alters the $\mathrm{pH}$ of the surrounding environment when it comes into contact with water or acidic solutions and this process exerts a significant effect on caries prevention [Nakatuka et al., 2003; Takada et al., 2007]. An optimal content of $20 \mathrm{wt} \% \mathrm{~S}-\mathrm{PRG}$ fillers in traditional resin denture bases was reported to provide sufficient fluoride release and recharge capacity in vitro and efficient inhibition of demineralization of bovine dentine while still maintaining acceptable strength in compliance with ISO 1567 [Kamijo et al., 2009; Mukai et al., 2009]. Despite the potential benefit that incorporation of S-PRG fillers in the resin denture base can provide to fitness and longevity of abutment teeth, very limited clinical data exist on fluoride release by denture base materials that incorporate S-PRG fillers. This clinical study aimed to examine the fluoride release and recharge capability of a dailyused partial resin denture containing 20wt\% S-PRG fillers and revealed that use of such a denture increased the saliva fluoride concentration in adults who wore it for 1.5 years. The null hypothesis was that no significant differences exist in the amount of fluoride released and the recharge of salivary fluoride between resin dentures with and without S-PRG fillers.

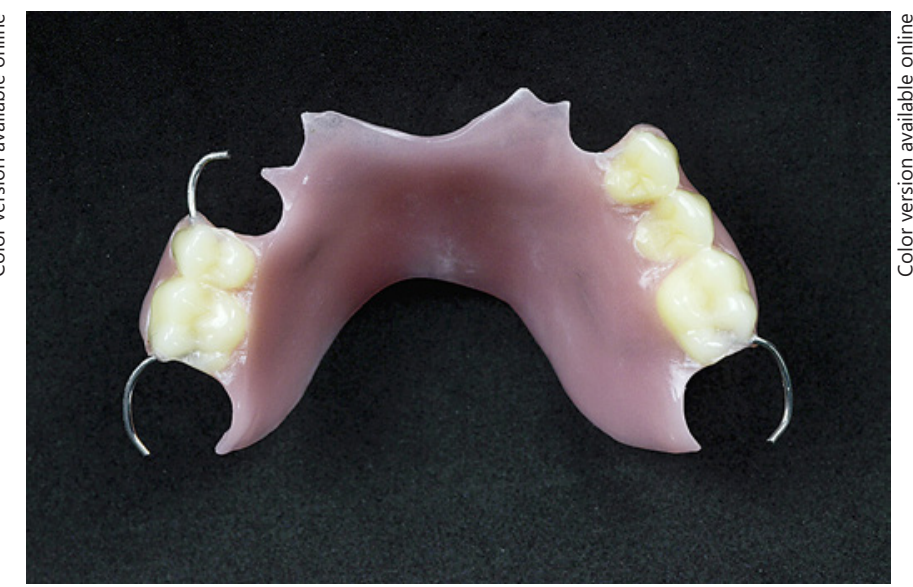

Fig. 2. Upper partial resin denture containing $20 \mathrm{wt} \% \mathrm{~S}-\mathrm{PRG}$ fillers.

\section{Methods}

This study was a 1.5-year double-blind, intervention-controlled clinical study and was reviewed and approved by the Institutional Review Board of the University of Washington, USA, and Thammasat University, Thailand, prior to implementation. A power calculation considered that the salivary fluoride concentration after the use of 1,000 ppm (parts per million) dentifrice remained at $0.02 \mathrm{ppm}$ at baseline [ten Cate, 2013], whereas the fluoride release from experimental resin dentures containing $20 \mathrm{wt} \%$ S-PRG filler on the first day after storage in distilled water was $0.006 \mathrm{mg} / \mathrm{mL}$ [Kamijo et al., 2009]. A difference in mean fluoride concentration in saliva between the control and the intervention group after wearing the denture on day 1 was expected to be approximately $0.04 \mathrm{ppm}$. A sample size of 45 subjects per group would be required to generate $80 \%$ power to detect this difference in mean saliva fluoride concentration using an independent $t$ test with two-sided 5\% statistical significance. Allowing for a possible drop-out rate of $20 \%$, an initial sample size of 110 subjects (55 per group) was recruited.

\section{Recruitment}

The enrollment of subjects occurred from June 2015 through September 2015 and follow-up took place from June 2015 through March 2017. Recruitment proceeded through local and hospital media at 3 government hospitals (Thammasat University Hospital, Kratumban Hospital, and Health Promotion Center Region 1 Hospital) in the vicinity of Bangkok, Thailand, where water fluoride content in the neighboring villages was less than $0.01 \mathrm{ppm}$. Approximately 35-40 subjects per hospital were randomly allocated into control and intervention treatment groups to receive an upper partial resin denture either without or with S-PRG filler. A block randomization was generated and coded as a numeric ID for each subject. The allocation list was provided to the dental assistant who assigned participants in sequential order to alternate treatment groups. The nature of group assignment was concealed from the participants and clinical staff until the conclusion of the study.

Healthy Thai patients aged 35-60 years who exhibited tooth loss and desire to have a resin denture fitted in their mouth were 


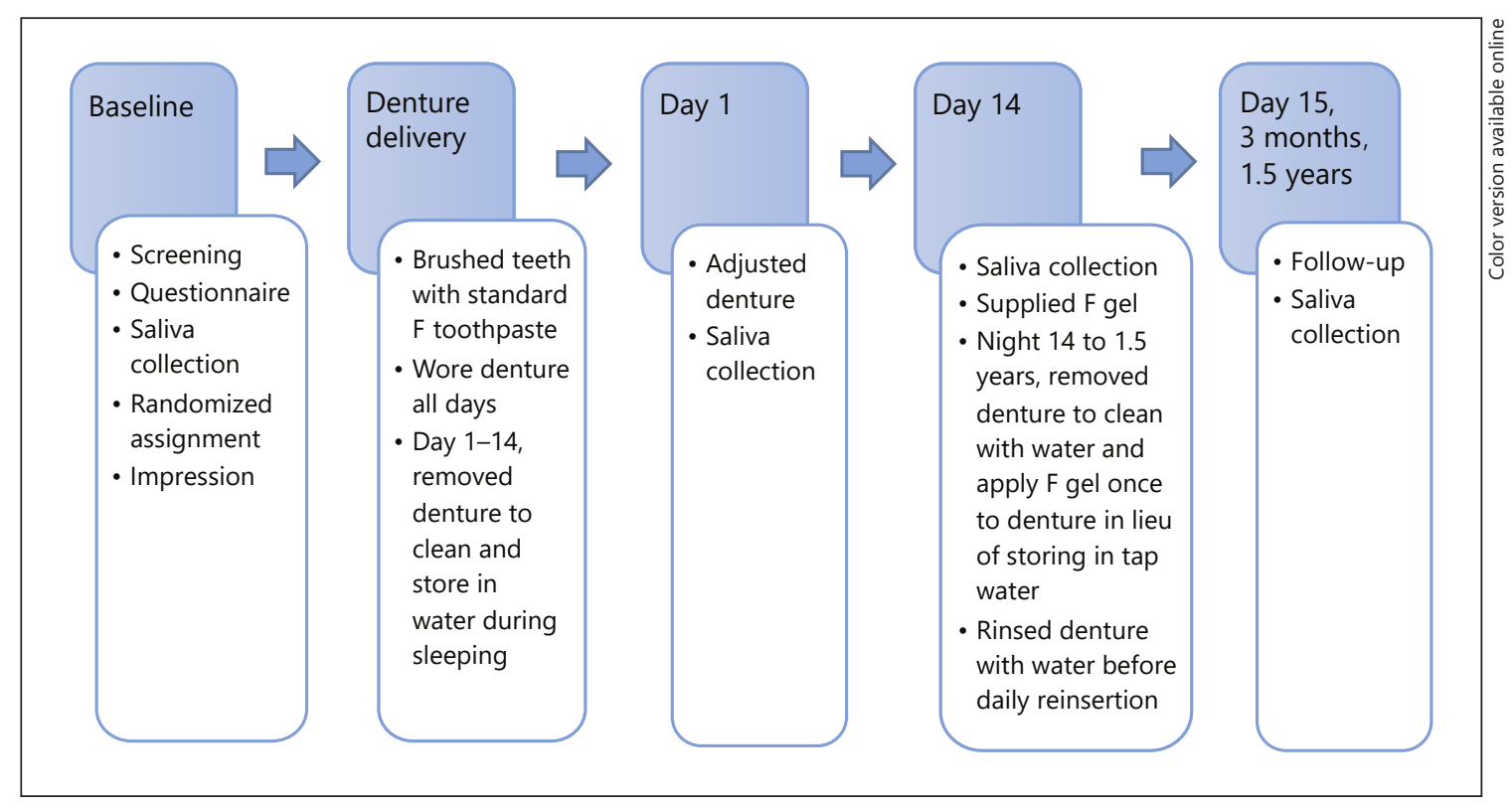

Fig. 3. Diagram of the procedure for partial resin denture wearers.

approached to participate in this study. At baseline, subjects completed a questionnaire of general demographic questions regarding medical health and oral care background, then received an initial oral screening and underwent a simple test of salivary flow rate by the participating dentist. Subjects who exhibited a salivary flow rate in the range of normal values (unstimulated whole saliva flow rate $>0.15 \mathrm{~mL} / \mathrm{min}$ ), and who retained at least 6 natural upper teeth, including at least 2 posterior teeth in good condition, were included in the study. Patients were excluded if they exhibited signs of xerostomia, had extensive dental caries or untreated periodontal disease, or had allergies to resin or metal that could interfere with the study. Information on study procedures was provided to subjects by participating dental assistants before written informed consent was obtained.

\section{Intervention}

Upper partial denture treatment was conducted by a participating dentist of the individual hospitals following standard protocols at baseline (Fig. 1, 2). After the models were poured and set, appropriate denture designs were drawn on the models and transcribed through generic lab script for communication with the laboratory that constructed the resin dentures according to their routine procedure. A notation on each script indicated to the laboratory whether the corresponding denture was to be made with polymethyl methacrylate resin (PMMA; URBAN RESIN ${ }^{\circledR}$, Shofu Inc., Kyoto, Japan) or with PMMA resin containing 20wt\% S-PRG fillers (URBAN RESIN ${ }^{\circledR}$ containing white S-PRG filler, particle size $4.1 \mu \mathrm{m}$, Lot 140122, Shofu Inc.). At the next appointment, dentures were fitted for their respective subjects and adjusted for comfortable wearing. To maintain blindness in the study, standard athome instructions for denture wearers were provided to all participants following denture delivery focusing on all days of wearing the denture, brushing the remaining teeth with a standard fluoride toothpaste (Colgate Total ${ }^{\circledR}, 1,000$ ppm; Colgate Palmolive, Thai- land), and cleaning the denture with water before storage in a denture storage box containing tap water while sleeping overnight for the first 14 days. After 14 days, 5,000 ppm fluoride gel (PreviDent ${ }^{\circledR}$ Gel, Colgate Palmolive) was supplied for all participants in both groups for storage of the dentures in lieu of tap water from night 14 to 1.5 years. Participants were instructed to remove the denture to clean it with water and apply $1 \mathrm{~g}$ of 5,000 ppm fluoride gel (approximately $1 \mathrm{~cm}$ of length) once on both sides before storage in the denture box overnight, repeating the procedure every night for 1.5 years. Participants were required to rinse the denture with water to remove the fluoride gel before daily reinsertion. A diagram of the full procedure for participants wearing the denture is given in Figure 3.

\section{Saliva Sample Collection}

At baseline, $2 \mathrm{~mL}$ of unstimulated saliva samples were collected from subjects before denture delivery and while wearing the denture on days $1,14,15$. This was repeated at 3 months and 1.5 years of wearing the denture, under the condition that the participants wore the denture for at least $1 \mathrm{~h}$ before and refrained from tooth brushing for at least $2 \mathrm{~h}$ before saliva sampling. All samples were kept frozen at $-80^{\circ} \mathrm{C}$ until fluoride analysis.

\section{Fluoride Analysis}

Samples were transferred to the stomatology laboratory, Prince of Songkhla University, Thailand, for the measurement of salivary fluoride content in duplicate, using the fluoride ion-selective electrode (ISE) direct method. Standard fluoride solutions with concentrations of 0.02 and $0.2 \mathrm{ppm}$ were used to calibrate the measuring device. Prior to measurement, $0.2 \mathrm{~mL}$ of total ionic strength adjustment buffer III (TISAB III; Cole-Palmer, USA) was added to each test solution, then a fluoride ion electrode (item number 27502-19, Cole-Palmer) was connected to a 710A-plus fluoride ion meter (item number 067952, Thermo Orion, USA). The fluoride 


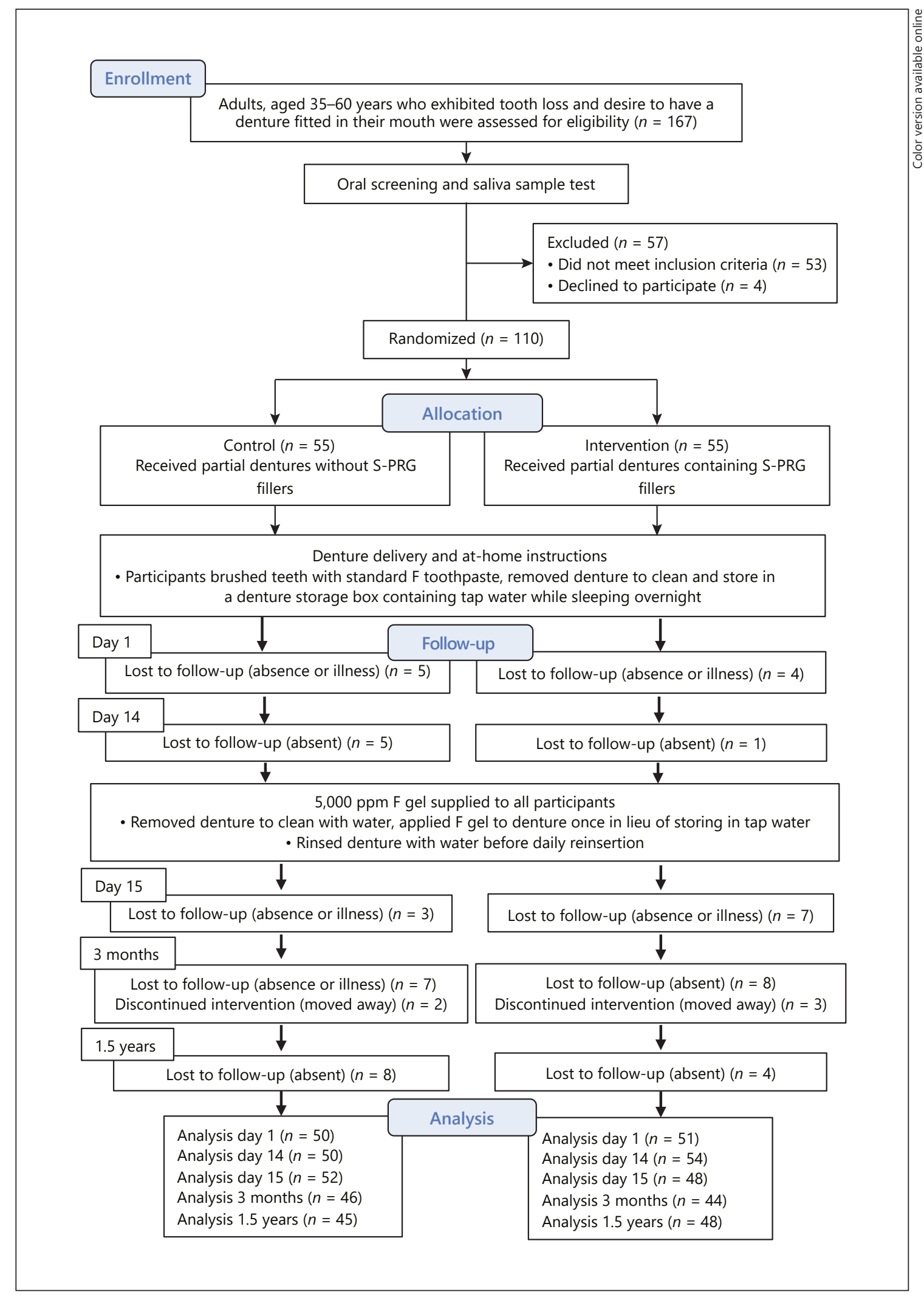

Fig. 4. Flow diagram of the study. 
concentration (ppm) was measured while the solution was stirred at room temperature. Two readings for each sample were recorded and the mean value was calculated. The samples were discarded after the analysis was completed.

\section{Statistical Analysis}

Data were analyzed with SPSS statistics software version 22.0 for Windows at $\alpha=0.05$. The statistical significance of observed differences in mean salivary fluoride concentrations (ppm) between the 2 treatment groups was assessed at different time points using repeated measures analysis of variance. The saliva fluoride concentrations from each group were statistically compared with the baseline concentration using a $t$ test.

\section{Results}

In total, 110 adults (24 males, 86 females) with a mean age of $49.7 \pm 10.4$ years fulfilled the study inclusion criteria and participated in this study. Participant drop-out rates of 5-20\% (by reason of absence or illness) were observed at each follow-up time point (see participant flow diagram, Fig. 4). No harm or adverse reaction was observed during the study. At baseline, the mean number of remaining natural teeth in the upper arch was 13 teeth for the control group and 12 teeth for the S-PRG filler group. Both groups had an average of 3 teeth replaced with artificial teeth on the upper partial resin denture. No significant differences in baseline demographics of the participants were observed between the 2 groups (Table 1). The mean concentration of saliva fluoride at baseline demonstrated no correlation with oral care characteristics of the participants, including frequency of tooth brushing and use of fluoride toothpaste and mouthwash ( $t$ test, $p>$ $0.05)$.

The mean salivary fluoride concentration (ppm) of both groups at the measured time intervals are given in Table 2 . The fluoride concentration increased significantly from baseline to day 1 of denture wearing in both groups, followed by a significant decrease to approximately baseline level by day $14(p<0.05)$. The mean salivary fluoride concentration was significantly higher for the S-PRG group compared to the controls on day 1 , but no difference between the 2 groups was observed at baseline or on day 14 . However, when the resin denture was recharged with 5,000 ppm fluoride gel, the mean salivary fluoride concentration in the test group increased significantly on day 15 , and at 3 months and 1.5 years, and was significantly greater than the control group, particularly after 3 months and 1.5 years. This result indicates that the differences in mean fluoride concentration of the intervention group were predominantly due to the recharging

Fluoride-Releasing Effect S-PRG Fillers

on Salivary Fluoride Retention
Table 1. Baseline demographics of the participants

\begin{tabular}{|c|c|c|c|}
\hline & $\begin{array}{l}\text { Control } \\
\text { denture }\end{array}$ & $\begin{array}{l}\text { S-PRG } \\
\text { denture }\end{array}$ & $\begin{array}{l}p \\
\text { value }\end{array}$ \\
\hline Participants, $n$ & 55 & 55 & \\
\hline Average age, years & $49.3 \pm 10.9$ & $49.8 \pm 10.1$ & 0.79 \\
\hline Gender, \% & & & 0.96 \\
\hline Male & 20.8 & 21.1 & \\
\hline Female & 79.2 & 78.9 & \\
\hline Teeth present & $12.4 \pm 1.9$ & $11.9 \pm 1.9$ & 0.11 \\
\hline Teeth replaced with denture & $2.4 \pm 1.5$ & $2.7 \pm 1.6$ & 0.26 \\
\hline Fluoride concentration, $\mathrm{ppm}$ & $0.05 \pm 0.04$ & $0.05 \pm 0.05$ & 0.38 \\
\hline Tooth brushing, $\%$ & & & 0.83 \\
\hline Twice a day & 58.5 & 75.4 & \\
\hline 3 or more times a day & 24.5 & 19.3 & \\
\hline Not identified & 17.3 & 5.3 & \\
\hline Fluoride toothpaste use, $\%$ & & & 0.15 \\
\hline Yes & 56.6 & 63.2 & \\
\hline No & 9.4 & 17.5 & \\
\hline Not identified & 34.0 & 19.3 & \\
\hline Fluoride mouthwash use, $\%$ & & & 0.07 \\
\hline Yes & 6.4 & 43.9 & \\
\hline No & 58.5 & 50.9 & \\
\hline Not identified & 15.1 & 5.3 & \\
\hline
\end{tabular}

Data are presented as the mean \pm SD or as indicated. $p$ values compare the baseline demographic of participants between the 2 groups.

with the 5,000-ppm fluoride gel daily from day 15 to 1.5 years. Although sustained fluoride release was observed in the control group after recharge with fluoride, the measured salivary fluoride concentration in this group began to decrease after 3 months, and was not significantly different from baseline concentration after 1.5 years. Fluoride concentrations in the control group were significantly lower than those of the experimental group at all of the time points assessed following nightly fluoride recharging.

Over 1.5 years of study, 7 subjects (4.6\%) experienced broken dentures, 2 of which were from the control group and 5 of which were from the S-PRG group ( $p=0.44$, Fisher exact test). These dentures were repaired or remade with materials according to the group assignment.

\section{Discussion}

Among denture-wearing patients, abutment teeth and teeth in contact with a removable denture are indicated as having a high caries risk [Featherstone et al., 2011]. The effect on caries risk of wearing a removable denture is 
Table 2. Salivary fluoride concentrations (ppm) over time

\begin{tabular}{lllllll}
\hline Group & Baseline & Day 1 & Day 14 & Day 15 & 3 months & 1.5 years \\
\hline Control & $0.05 \pm 0.04$ & $<0.09 \pm 0.07^{\mathrm{a}}$ & $0.06 \pm 0.06$ & $<0.11 \pm 0.11^{\mathrm{a}}$ & $<0.12 \pm 0.12^{\mathrm{a}}$ & $<0.08 \pm 0.12$ \\
S-PRG & $0.05 \pm 0.05$ & $<0.22 \pm 0.12^{\mathrm{a}}$ & $0.06 \pm 0.05$ & $<0.23 \pm 0.13^{\mathrm{a}}$ & $<0.26 \pm 0.15^{\mathrm{a}}$ & $<0.32 \pm 0.20^{\mathrm{a}}$ \\
$p$ value & 0.658 & $<0.001$ & 0.733 & $<0.001$ & $<0.001$ & $<0.001$ \\
\hline
\end{tabular}

Values are presented as the mean \pm SD. $p$ values indicate the level of significant difference in salivary fluoride concentration between the test and control groups at each time point. ${ }^{\text {a }}$ Indicates a significant difference in salivary fluoride concentration compared with the baseline concentration within the same group $(p<0.05)$.

controversial, but it may relate to the difficulty with access for cleaning or with increased plaque accumulation and risk of caries seen with the use of the dentures [Bergman et al., 1995]. To prevent dental caries affecting abutment teeth associated with removable dentures, fluoride delivery into the oral cavity is recommended. One possible mode of fluoride delivery is through the use of a fluoridated denture. In the present study, 20wt\% S-PRG fillers were incorporated into a resin denture base and acted as a fluoride source. The resin dentures containing S-PRG were initially capable of fluoride release in a burst effect for 1 day of wearing the denture, which is a known and unique property of fluoride-containing materials [Dhondt et al., 2001]. The dentures exhibited a fluoridereleasing phenomenon consistent with that observed previously in vitro [Wiegand et al., 2007; Kamijo et al., 2009]. However, the amount of initial fluoride release from within the dentures was reduced to baseline levels after 14 days. This observation indicated that S-PRG-containing dentures led to an elevation of fluoride in the oral cavity, but the initial effect was short lived, consistent with expectations due to the kinetics of saliva fluoride clearance [ten Cate, 2013].

While the benefit of the initial burst lies in fluoride's proven effect in caries prevention and the remineralization of dental tissue, a sustained release of fluoride is associated with long-term anticariogenic action [Itota et al., 2004b]. A constant supply of low levels of intraoral fluoride from 0.03 to $0.1-0.3 \mathrm{ppm}$ (i.e., a 5 - to 10 -fold increase) for prolonged periods is of most benefit in caries control [Duckworth and Morgan, 1991; Sjogren and Birkhed, 1993]. Importantly, S-PRG fillers have been reported to exhibit a fluoride-releasing capacity after recharge [Zafar, 2013; Dionysopoulosa, 2014]. The S-PRGcontaining denture in this study exhibited a fluoride-recharging capacity that appeared to consistently maintain fluoride release following overnight recharge with 5,000 ppm fluoride gel daily from day 15 through to 1.5 years.
The potential for a S-PRG-containing resin denture to maintain a constant release of fluoride, thereby increasing the fluoride level in the oral environment whenever the denture is frequently recharged with fluoride, coupled with the observed salivary fluoride concentration of $0.2-$ $0.3 \mathrm{ppm}$ for this type of denture, has the potential to control caries on abutment teeth in contact with the denture.

The degree of fluoride-recharging ability from fluoridated resin depends on many factors, including the level of fluoride content, kind and frequency of fluoride exposure, and concentration of the fluoridating agent [Han et al., 2002; Toumba and Curzon, 2005]. In this study, recharge of the resin denture base containing S-PRG fillers provided an optimal salivary fluoride level after daily recharge with 5,000 ppm fluoride gel. This finding is consistent with a previous investigation that reported the ability of fluoridated glass filler within a denture base resin to facilitate fluoride release following recharge with 5,000 ppm NaF gel [Al-Bakri et al., 2014]. Thus, the use of a 5,000-ppm fluoride concentration for recharge of fluoride may be realistic for clinical use. However, 5,000 pm fluoride gel is available by prescription only and, given that it is more expensive than regular fluoridated dentifrices $(1,100 \mathrm{ppm})$, some patients may reject the idea of applying it. A regular fluoridated dentifrice may be an alternative in such cases.

The mechanism of fluoride release from the S-PRGcontaining denture base observed in this study is related to the presence of glass-ionomer phase around the glass core of the filler [Fujimoto et al., 2010]. As the S-PRG particle is a prereacted glass ionomer filler in a resin matrix, its fluoro-aluminosilicate glass has been prereacted with polyacid to form a glass-ionomer matrix structure which is then blended with PMMA resin. An almost complete acid-base reaction has already taken place before incorporation into the resin, therefore this glass-ionomer matrix contains much complex fluoride and is easily penetrated by water, which results in significant fluoride re- 
lease from the material at the initial stage [Najma Hajira and Meena, 2015]. Although the precise effect of fluoride release following the recharge of the denture is not fully understood, it is acknowledged that the recharge ability is dependent on the extent of the hydrogel matrix within the S-PRG particles, which serve as a reservoir for storage of fluoride and a vehicle for continuous release [Itota et al., 2004b]. Furthermore, PMMA denture base material, which is hydrophilic in nature, may absorb the solution and play a role in the dissolution of fluoridated glass fillers, resulting in an increase of fluoride uptake and release.

By comparison, a similar pattern of fluoride release was observed for the control dentures as for the dentures containing S-PRG fillers, but the amount of salivary fluoride measured was significantly lower than that for a denture containing S-PRG fillers, and was not maintained following recharge for the entire 1.5 years of study. The reduced effect following the initial release of fluoride from the control resin implies that only the fluoride adsorbed to the surface topography of the control denture was released. This fluoride release from the control denture may result from the physical properties of PMMA resins that are subjected to water sorption when placed in an aqueous environment [Cunha et al., 2009]. These resins absorb fluoride from the oral environment to the surface of the material and sustain fluoride release in the manner of fluoridated materials. Additionally, chemical reactivity of PMMA resin may be implicated in the form of the remaining unreacted monomer in the denture base. Residual monomer may be leached and affected by bulk sites of material that facilitate the diffusion of salivary fluoride toward the external surface. However, once the bulk sites are fully occupied, their permeability may be limited, resulting in a decrease of fluoride concentration, as observed in this study [Bhola and Mishra, 2010]

In the present study, some patients experienced broken dentures due to accidents such as dropping the denture or biting when the denture was not correctly in position. Broken dentures comprised less than $5 \%$ of the total dentures placed and no significant difference in the incidence of broken dentures was observed between groups. Resin denture base containing 20wt\% S-PRG filler did not appear to diminish the mechanical properties of the denture over the study period of 1.5 years. This concentration may be an optimal percentage of filler that satisfies the need for fluoride release while maintaining acceptable strength compliant with the requirement of ISO1567 [Kamijo et al., 2009].

An advantage of this study was that almost all participants were approached from the waiting list for remov- able partial dentures of each hospital, meaning the oral status of participants had been previously prepared for denture fabrication. Although more than 3 times the number of female participants were recruited than male participants (86 females and 24 males), no differences in performance were found between female and male participants. Participants in this study were followed with a similar intervention as that for routine denture wearers. The difference between these groups was in the materials used for the fabrication of their dentures. However, the dentures fabricated with and without S-PRG fillers were so similar in appearance and in color that they were difficult to distinguish visually.

A limitation was in relation to the ISE method used to determine saliva fluoride concentration in the study. This method requires TISAB buffer solution to control $\mathrm{pH}$ and this ensures that the total fluoride ion concentration (free and complex fluoride) can be determined. Although the ISE with TISAB III is simple, cost effective, and convenient for the measurement of fluoride release, the method is limited by the uncertainty of measuring at low fluoride concentrations, particularly given the difficulties of achieving a stable meter reading with no drift. The lower limit of fluoride determination with the electrode is 0.1 $\mathrm{ppm}$. Below this concentration, the output from the electrode becomes meaningless [McCabe et al., 2002]. To overcome the shortcomings with ISEs, ion chromatography is a useful alternative method for fluoride determination at very low concentrations because of its high sensitivity, which provides meaningful and reproducible measurements [Itota et al., 2004a].

This study demonstrated that sustained release of fluoride from the resin denture containing S-PRG filler through the recharging route was significantly greater than that released from within the material, and is expected to have the long-term effect of increasing enamel and dentin resistance to new carious lesions on abutment teeth of partial denture wearers. The capacity of a fluoridated resin denture base to act as a fluoride reservoir may be an important consideration in the selection of materials for clinical use in the near future. However, further investigation should reveal the efficacy of this material for the prevention of new carious lesions and also should focus on facilitation of incorporation of a higher content of S-PRG filler, which would satisfy the financial need of patients for fluoride recharge using the lowest and therefore least expensive possible fluoride concentration recharge material.

Within the limitations of the study, we can conclude that the resin denture base containing 20wt $\%$ S-PRG fillers demonstrated initial fluoride release to increase saliva 
fluoride concentrations, but the fluoride release from within the material was of short duration. The long-term effect of sustained fluoride release from the denture requires combination with a regular fluoride recharge regimen, which in turn is expected to act as a fluoride reservoir for the controlling of dental caries on abutment teeth of partial denture wearers.

\section{Acknowledgements}

The authors acknowledge Dr. Timothy A. DeRouen, Professor Emeritus, Biostatistics, Oral Health Science, and Global Health, University of Washington, for his comments and statistical advice. PMMA resin and S-PRG fillers were kindly provided by Shofu Inc. Kyoto, Japan. This study was supported by the Fogarty Interna- tional Center (FIC) and the National Institute of Dental and Craniofacial Research (NIDCR) of the National Institutes of Health under Award No. D43TW009071. The funders had no role in study design, data collection and analysis, decision to publish, or preparation of the manuscript.

\section{Disclosure Statement}

The authors declare no conflicts of interest.

\section{Author Contributions}

Study conception and design: K.K., D.C.N.C.; performance of clinical experiments: K.K., D.C.N.C.; data analysis: K.K., T.S., P.P.; manuscript preparation: K.K., T.S., P.P., D.C.N.C.

\section{References}

Al-Bakri IA, Swain MV, Naoum SJ, Al-Omari WM, Martin E, Ellakwa A: Fluoride release, recharge and flexural properties of polymethylmethacrylate containing fluoridated glass fillers. Aust Dent J 2014;59:208-214.

Bergman B, Hugoson A, Olsson CO: A 25 year longitudinal study of patients treated with removable partial dentures. J Oral Rehabil 1995; 22:595-599.

Bhola R, Bhola SM, Liang H, Mishra B: Biocompatible denture polymers - a review. Trends Biomater Artif Organs 2010;23:129-136.

Cunha TR, Regis RR, Bonatti MR, de Souza RF: Influence of incorporation of fluoroalkyl methacrylates on roughness and flexural strength of a denture base acrylic resin. J Appl Oral Sci 2009;17:103-107.

Dhondt CL, De Maeyer EA, Verbeeck RM: Fluoride release from glass ionomer activated with fluoride solutions. J Dent Res 2001;80:14021406.

Dionysopoulosa D: The effect of fluoride-releasing restorative materials on inhibition of secondary caries formation. Fluoride 2014;47: 258-265.

Duckworth RM, Morgan SN: Oral fluoride retention after use of fluoride dentifrices. Caries Res 1991;25:123-129.

Featherstone JD, Singh S, Curtis DA: Caries risk assessment and management for the prosthodontic patient. J Prosthodont 2011;20:2-9.
Fujimoto Y, Iwasa M, Murayama R, Miyazaki M, Nagafuji A, Nakatsuka T: Detection of ions released from S-PRG fillers and their modulation effect. Dent Mater J 2010;29:392-397.

Han L, Cv E, Li M, Niwano K, Ab N, Okamoto A, Honda N, Iwaku M: Effect of fluoride mouth rinse on fluoride releasing and recharging from aesthetic dental materials. Dent Mater J 2002;21:285-295.

Itota T, Carrick TE, Rusby S, Al-Naimi OT, Yoshiyama M, McCabe JF: Determination of fluoride ions released from resin-based dental materials using ion-selective electrode and ion chromatograph. J Dent 2004a;32:117122.

Itota T, Carrick TE, Yoshiyama M, McCabe JF: Fluoride release and recharge in giomer, compomer and resin composite. Dent Mater J 2004b;20:789-795.

Kamijo K, Mukai Y, Tominaga T, Iwaya I, Fujino F, Hirata Y, Teranaka T: Fluoride release and recharge characteristics of denture base resins containing surface pre-reacted glass-ionomer filler. Dent Mater J 2009;28:227-233.

McCabe JF, Carrick TE, Sidhu SK: Determining low levels of fluoride released from resin based dental materials. Eur J Oral Sci 2002; 110:380-384.

Mukai Y, Kamijo K, Fujino F, Hirata Y, Teranaka T, ten Cate JM: Effect of denture base-resin with prereacted glass-ionomer filler on dentin demineralization. Eur J Oral Sci 2009;117: 750-754.
Najma Hajira NSW, Meena N: GIOMER - the intelligent particle (new generation glass ionomer cement). Int J Dent Oral Health 2015, DOI: $10.16966 / 23787090.166$.

Nakatuka T, Yasuda Y, Kimoto K, Mizuno M, Negoro N: Dental fillers. US Patent Application 6620861 B1. 2003.

Sjogren K, Birkhed D: Factors related to fluoride retention after toothbrushing and possible connection to caries activity. Caries Res 1993; $27: 474-477$.

Takada T, Morigami M, Sugizaki S, Uno S, Yamada T: Improvement of giomer compositeSEM observation and clinical application of Beautifil II. Adhes Dent 2007;25:19-23.

ten Cate JM: Contemporary perspective on the use of fluoride products in caries prevention. Br Dent J 2013;214:161-167.

Toumba KJ, Curzon ME: A clinical trial of a slowreleasing fluoride device in children. Caries Res 2005;39:195-200.

Wiegand A, Buchalla W, Attin T: Review on fluoride-releasing restorative materials-fluoride release and uptake characteristics, antibacterial activity and influence on caries formation. Dent Mater J 2007;23:343-362.

Zafar MS: Effects of surface pre-reacted glass particles on fluoride release of dental restorative materials. World Appl Sci J 2013;28:457-462. 